Notre Dame Journal of Formal Logic

Volume XIX, Number 3, July 1978

NDJFAM

\title{
THE MINIMAL MODAL LOGIC: A CAUTIONARY TALE ABOUT PRIMITIVES AND DEFINITIONS
}

\section{MARILYN MILBERGER}

* On page 254 of [2], Hughes and Cresswell list several weak adequacy criteria for propositional modal logics. One is that

a) $\sim \square \sim p \equiv \diamond p$

and

b) $\sim \diamond \sim p \equiv \square p$

be theses. On page 255 they say that the weakest logic satisfying their conditions is the S0.5 of [3]. A formulation of this logic is given in which the square is the only primitive modal operator, and they add that "the other modal operators are defined so as to satisfy" the condition given and similar requirements for strict implication and strict coimplication. As for the sense of necessity represented by the S0.5 square, they quote Lemmon, in [4], as proposing that it be read "it is tautologous by truth tables that. ...'

It would seem that, as they present S0.5,

c) $\square(\sim \diamond p \equiv \square \sim p)$

is a thesis, but

d) $\square(\sim \square p \equiv \diamond \sim p)$

is not. Why? Since the diamond is introduced by definition, (c) amounts to

e) $\square(\sim \sim \sim p \equiv \square \sim p)$

which is the necessitate of

f) $(\sim \sim \square \sim p \equiv \square \sim p)$

*This paper was written as a result of conversations with Jay Hartman and correspondence with Robert Purdy. 
which is obviously truth-functionally valid. In contrast, (d) reduces to the necessitation of

g) $(\sim \square p \equiv \sim \square \sim \sim p)$

which is not a truth-table tautology since the double negation on one side of the triple bar is inside the scope of a necessity operator.

One supposes that, had S0.5 been formulated with the diamond as primitive and the square introduced by definition, (d) would have been a thesis and (c) not. Further, one can define a system with the same motivating intuitive interpretation of the modal operators, but taking both necessity and possibility as primitive, in which neither (c) nor (d) is a thesis. It is easy to specify the set of theses of such a system. It is the smallest set containing all valid first-degree modal formulas ${ }^{1}$ and closed under uniform substitution of formulas for sentence letters.

This logic, which I would like to name I (for Infimum), can be given a natural deduction formulation in the manner of (1) by adding to a formulation of the two-valued propositional calculus the following rules:

i) a formula is a direct consequence of its own necessitate, and its possibilitate is a consequence of it,

ii) the negation of the necessitate (possibilitate) of a formula is a direct consequence of the possibilitate (necessitate) of the negation of that formula, and vice versa,

iii) the necessitate of a formula is a direct consequence of a strict subordinate proof with no hypotheses and containing it as an item,

iv) the possibilitate of a formula is a direct consequence of the possibilitate of a second together with a strict subordinate proof having the second formula as its only hypothesis and containing the first formula as an item,

under the restrictions that

$\mathrm{x}$ ) only necessitates may be reiterated into strict subordinate proofs, and they drop their initial necessity operator as they go, ${ }^{2}$

y) none of the modal rules may be used within a strict subordinate proof.

Proof of the soundness and completeness of the natural deduction system relative to the specification of theses in terms of first degree validity and substitution is easy, and it is obvious that the natural deduction system suffices to prove anything provable from the "official" rules and axioms of S0.5 given on page 256 of [2]. ${ }^{3}$

What, then, is the weakest logic fulfilling the various conditions Hughes and Cresswell give? The set of theses derivable from the official rules and axioms of S0.5 is a proper subset of the set of theses of I: it includes no formulas containing occurrences of the possibility symbol. In particular, it does not include (a) or (b). "Official" s0.5, therefore, does not 
fulfill the conditions listed. Hughes and Cresswell think of S0.5 as qualifying, so "practical" s0.5 must contain, in addition to the official rules and axioms, a "rewrite" rule of the form

r) any formula obtained from a thesis by replacing one or more occurrences of

with occurrences of

is also a thesis. But then "practical" S0.5 has (c) as a thesis, and so is strictly stronger than I.

\section{NOTES}

1. That is, first-degree formulas which are theses of any of the usual modal logics. These logics have a common first-degree fragment, which can well be thought of as absolute, for it is justified by very weak assumptions about modality and, given uniform substitution, cannot be strengthened on pain of inconsistency, modal collapse, or finite characteristic matrix.

2. Imposing this restriction without (y) would get you the system $T$ of Feys and Von Wright, described in chapter 2 of [2] as the weakest logic fulfilling another set of conditions.

3. On the other hand, the reading of the square as "it is tautologous by truth tables that..." must be taken with a grain of salt, here and in S0.5. No necessitate is truth functionally valid, but

$$
\square \square
$$

is not refutable.

\section{REFERENCES}

[1] Fitch, F. B., Symbolic Logic, an Introduction, New York, Ronald Press (1952).

[2] Hughes, G. E., and M. J. Cresswell, An Introduction to Modal Logic, London, Methuen (1968).

[3] Lemmon, E. J., "New foundations for Lewis modal systems," The Journal of Symbolic Logic, vol. 22 (1957).

[4] Lemmon, E. J., "Is there only one correct system of modal logic?", Aristotelian Society Supplementary, vol. XXXIII (1959).

University of Pittsburgh

Pittsburgh, Pennsylvania 